\title{
Plasmonic nanogels with robustly tunable optical properties
}

\author{
Tao Cong ${ }^{a}$, Satvik N. Wani ${ }^{\mathrm{a}}$, Georo Zhou ${ }^{\mathrm{a}}$, Elia Baszczuk ${ }^{\mathrm{a}}$, Radhakrishna Sureshkumar*a, b \\ ${ }^{a}$ Department of Biomedical and Chemical Engineering, Syracuse University, Syracuse, NY USA \\ 13244 \\ ${ }^{\mathrm{b}}$ Department of Physics, Syracuse University, Syracuse, NY USA 13244
}

\begin{abstract}
Low viscosity fluids with tunable optical properties can be processed to manufacture thin film and interfaces for molecular detection, light trapping in photovoltaics and reconfigurable optofluidic devices. In this work, self-assembly in wormlike micelle solutions is used to uniformly distribute various metallic nanoparticles to produce stable suspensions with localized, multiple wavelength or broad-band optical properties. Their spectral response can be robustly modified by varying the species, concentration, size and/or shape of the nanoparticles. Structure, rheology and optical properties of these plasmonic nanogels as well as their potential applications to efficient photovoltaics design are discussed.
\end{abstract}

Keywords: Plasmonics, Self-assembly, Optofluidic, Photovoltaic, Surfactant micelle, Nanotechnology, Solar Energy

\section{INTRODUCTION}

Localized surface plasmons that can be excited in noble metal nanostructures ${ }^{1,2}$ have potential applications in the broadband manipulation of light at the nanoscale. ${ }^{3}$ The surface plasmon resonance occurs in the visible region of the electromagnetic spectrum for plasmonic nanoparticles (NPs) that are made of noble metals such as $\mathrm{Ag}, \mathrm{Au}$ and $\mathrm{Cu}^{1,4,5}$ The strong size and shape-dependence of the optical extinction spectrum gives rise to a variety of colors to suspensions of such NPs. ${ }^{4}$ NPs with lower shape symmetry can support more plasmon modes and hence possess a broader extinction spectrum. ${ }^{6}$ Nanorods, for example, have two resonances corresponding to the axial and longitudinal dimensions. ${ }^{7,8}$ Hence, complex shapes such as nanocages, nanoprisms and aggregates have a relatively broader extinction and scattering spectrum. ${ }^{6,9}$ The tunability of plasmonic properties is highly desirable for many applications that rely on nanoscale light manipulation such as surface-enhanced Raman scattering (SERS) ${ }^{10}$ sensing, ${ }^{11}$ imaging, ${ }^{12}$ therapeutics, ${ }^{13}$ photocatalysis, light harvesting ${ }^{14,15}$ and optofluidics. ${ }^{16,17}$ The strong interaction of metal NPs with the optical field also allows for light trapping that can be used for efficient photovoltaic design. ${ }^{18}$

Optofluidics refers to a class of optical systems that are synthesized with fluids, which have unique properties that cannot be found in solid equivalents. ${ }^{17}$ The advantages include the ability to change the optical property of the fluid medium within a device by replacing one fluid with another, creation of optically smooth interfaces between two immiscible fluids and the ability of flowing streams of miscible fluids to create gradients in optical properties by diffusion. ${ }^{17}$ Growth in this area has been driven by the progress in the modeling and handling of fluid systems at micro to nanometer scale, which leverages the fabrication techniques originally developed for integrated circuits and microelectromechanical systems. NP suspensions offer important advantages to optofluidic systems since the nanoparticle type, shape and concentration can be used to tailor the optical properties of the suspension. ${ }^{19}$ The design of multicomponent NP suspensions can be guided by effective medium theories for the dielectric function of the composite material. ${ }^{2021}$

Typically, metal nanoparticles in a solution tend to agglomerate. This often leads to phase separation. In this work, we used a network of aqueous wormlike surfactant micelles (WLMs) as a template for producing stable multicomponent suspensions of $\mathrm{Au}$ and/or $\mathrm{Ag}$, hereafter referred to as plasmonic nanogels (PNGs). Cetytrimethylammonium bromide (CTAB) is one of the most well-studied wormlike micelle (WLM) forming surfactants. ${ }^{22}$ Wormlike micelles are elongated and semiflexible cylindrical aggregates formed by the self-assembly of amphiphilic surfactants in aqueous solutions. At sufficiently large concentrations, the micellar chains entangle to form a viscoelastic network with linear dimension on the order of $\left(k_{\mathrm{b}} T / G_{0}\right)^{1 / 3}$ where $G_{0}$ is the plateau storage modulus and $k_{\mathrm{b}}$ and $T$ represent the Boltzmann constant and absolute temperature respectively. The headgroup of the cationic surfactant molecules can bind to the surface of the metal nanoparticles as suggested by Fourier transform infrared spectroscopy and thermogravimetric analysis. ${ }^{23}$ Colloidal particles in a WLM solution are known to form a network consisting of particle-micelle junctions. ${ }^{24}$,

Optical Trapping and Optical Micromanipulation VIII, edited by Kishan Dholakia, Gabriel C. Spalding, Proc. of SPIE Vol. 8097, 80970L · C 2011 SPIE · CCC code: 0277-786X/11/\$18 · doi: 10.1117/12.894070 
${ }^{25}$ Such networks are capable of distributing nanoparticles with different sizes and shapes within the fluid in a stable and uniform fashion.

Our design of the plasmonic nanogels was based on the existing knowledge of plasmon resonance in small particles. ${ }^{5}$ Specifically, the plasmon resonance in particles that are much smaller than the wavelength of light can be modeled accurately by Maxwell equations under the quasistatic approximation. ${ }^{26}$ The polarizability, $\alpha$, of a spherical particle is given by eq. 1 below. ${ }^{5}$

$$
\alpha=4 \pi a^{3} \frac{\varepsilon_{p}-\varepsilon_{m}}{\varepsilon_{p}+2 \varepsilon_{m}} .
$$

Here, $\varepsilon_{\mathrm{p}}$ and $\varepsilon_{\mathrm{m}}$ are the relative permittivities of the particle and surrounding medium respectively and $a$ is the particle radius. Resonance occurs when the denominator in eq. 1 approaches zero, i.e. $\varepsilon_{p}=-2 \varepsilon_{m}$. However, for larger particles, higher order terms that correspond to the electrical and magnetic multipoles in Mie series solution need to be considered. $^{5}$ The resonance wavelength shifts to the red region for relatively larger particles because the Coulombic coupling of the regions of positive and negative surface charge becomes relaxed at larger diameters. The factor of 2 in the denominator changes for small non-spherical particles. ${ }^{4,5}$ Based on this understanding of the polarizability of nanoparticles of various materials and shapes, it was possible to design PNGs that possessed a desired optical extinction spectrum.

\section{ONE-COMPONENT PLASMONIC NANOGELS}

CTAB WLMs were prepared by vortex mixing CTAB powder with deionized water to the desired final concentrations, followed by growth under $50{ }^{\circ} \mathrm{C}$ for 1 hour. Plasmonic nanogels (PNGs) were made by the addition of nanoparticles NPs (Au NPs: Nanopartz, Inc.; Ag NPs: Sigma-Aldrich) to the stable WLMs under agitation. All samples were equilibrated at $24{ }^{\circ} \mathrm{C}$ overnight prior to measurements. The extinction spectrum of PNGs with gold nanospheres (S) is shown in Figure. 1 . The resonance peak redshifts from $\approx 520 \mathrm{~nm}$ to $\approx 580 \mathrm{~nm}$ as particle size increases from $30 \mathrm{~nm}$ to $90 \mathrm{~nm}$. The extinction of small particles is dominated by the absorption. Scattering dominates over absorption for large particles. ${ }^{5}$

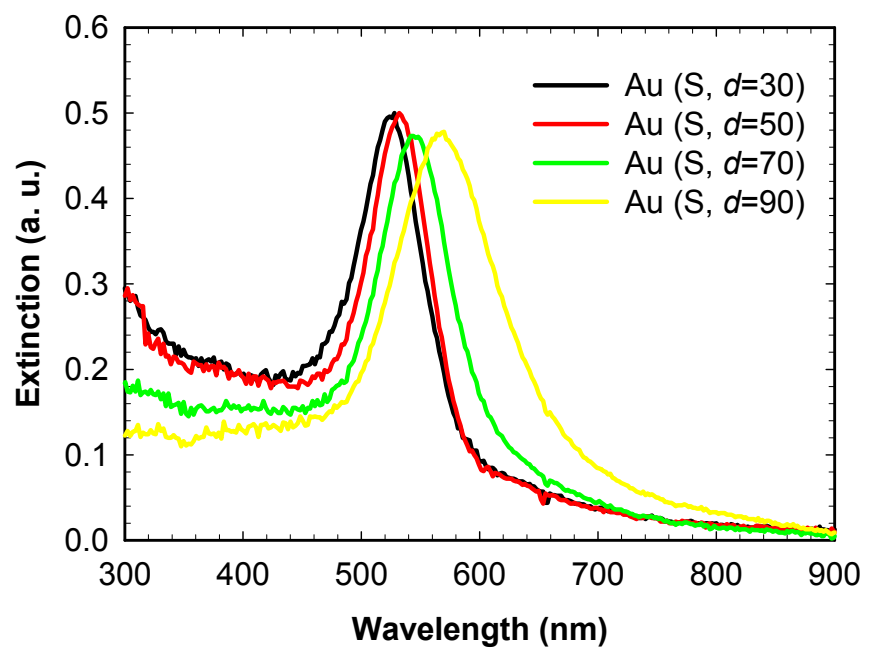

Figure 1. Redshift of the plasmonic resonance peak of Au nanogels with increasing diameter. The curves show extinction spectra for particle diameters of 30 (black), 50 (red), 70 (green) and $90 \mathrm{~nm}$ (yellow).

The refractive index of the NPs depends on both material composition and the particle shape. ${ }^{27}$ So, the peak extinction wavelength is also strongly dependent on the shape of the NPs. NPs can be produced in a variety of shapes as well, including sphere, rod, cube, triangle/prism, hexagon, star, etc. For non-spherical particles, multiple resonances will appear. For instance, gold nanorods have two extinction peaks, which correspond to the transverse (short axis) plasmon resonance and longitudinal (long axis) plasmon resonance. The crucial factors determining the position of the extinction 
peaks of gold nanorods are their aspect ratio and the radial dimension. All the nanorods in Figure 2 have a diameter of $\approx$ $25 \mathrm{~nm}$. So, by increasing the aspect ratio, the longitudinal absorption peak redshifts to the near-infrared region as shown in Figure 2. For NPs of more complex structures, numerical methods are used to study and predict the plasmon resonance spectrum, such as finite difference time domain (FDTD) and finite element method (FEM).

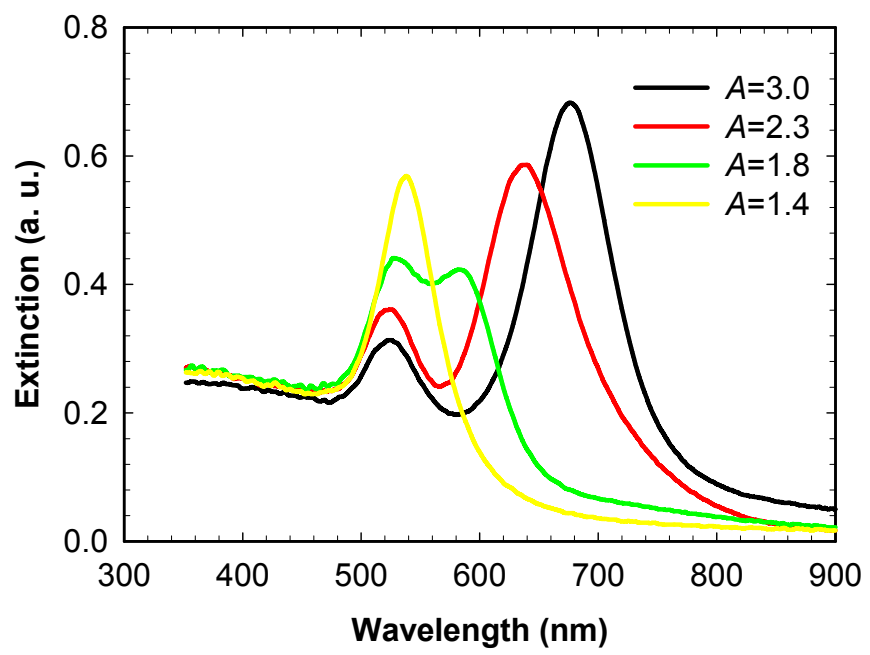

Figure 2. Extinction spectra of gold nanorods (diameter $\approx 25 \mathrm{~nm}$ ) with aspect ratio $(A)$ ranging from 1.4 to 3.0. Nanorod with an aspect ratio of 1.4 shows one resonance peak (black) while rods with larger aspect ratios show two peaks (red, green and yellow).

\section{MULTICOMPONENT PLASMONIC NANOGEL}

NPs of various materials, sizes and shapes exhibit different extinction spectra due to the differences in their polarizability. Extinction increases linearly with concentration for relatively low nanoparticle concentrations. ${ }^{5}$ The addition of multiple species of NPs resulted in an extinction spectrum, which is a linear superposition of the individual spectra. Au nanosphere, Ag nanosphere and Au nanorod exhibited red, yellow and blue colors respectively. Various colors could be produced by suitably mixing metal NPs exhibiting those three primary colors. As shown in Figure 3, PNGs consisting of different types of NPs provided a fascinating range of colors. Small angle X-ray scattering experiments suggest that the nanoparticles are uniformly distributed in the micelle matrix without agglomeration. ${ }^{28}$ The optical property of each sample was examined by UV-Vis-spectrometry. The multicomponent PNGs showed multiple plasmon resonance peaks in the visible light to near-infrared region. For example, sample 5 (green curve in Figure 3) showed three absorption peaks at $\approx 420 \mathrm{~nm}, \approx 520 \mathrm{~nm}$ and $\approx 640 \mathrm{~nm}$. The first peak is due to the plasmon resonance of $\mathrm{Ag}$ nanospheres while the other two peaks were attributed to the transverse and longitudinal plasmon resonance of $\mathrm{Au}$ nanorod. This offers a new approach to tune the optical property of optical fluids. Specifically, instead of synthesizing nanoparticles in complex geometries, such as crescent moon structures ${ }^{29}$ star-shaped gold nanoparticle,${ }^{30}$ one can produce nanomaterials with desired optical properties by suitably mixing nanoparticles that can be easily synthesized.

Light trapping can be achieved in a wide range of wavelength by changing NPs types and geometries. Sample 8 with five nanoparticle species (as listed in the Figure 4) were prepared to obtain a more uniform extinction spectrum. Upon tuning the fraction of each component in the nanogel, the multicomponent PNG showed a broadband absorption capability. As shown in Figure 4, sample 8 exhibited four peaks at $\approx 420 \mathrm{~nm}, \approx 520 \mathrm{~nm}, \approx 640 \mathrm{~nm}$ and $\approx 750 \mathrm{~nm}$. The last peak is due to the addition of gold nanorod with a larger aspect ratio of 3.5. Such PNG samples have potential application as a simple and direct method of optimizing light trapping layers in photovoltaic devices. In addition, due to their tunable optical absorption, PNGs can be potentially used in photothermolysis. ${ }^{31}$ 


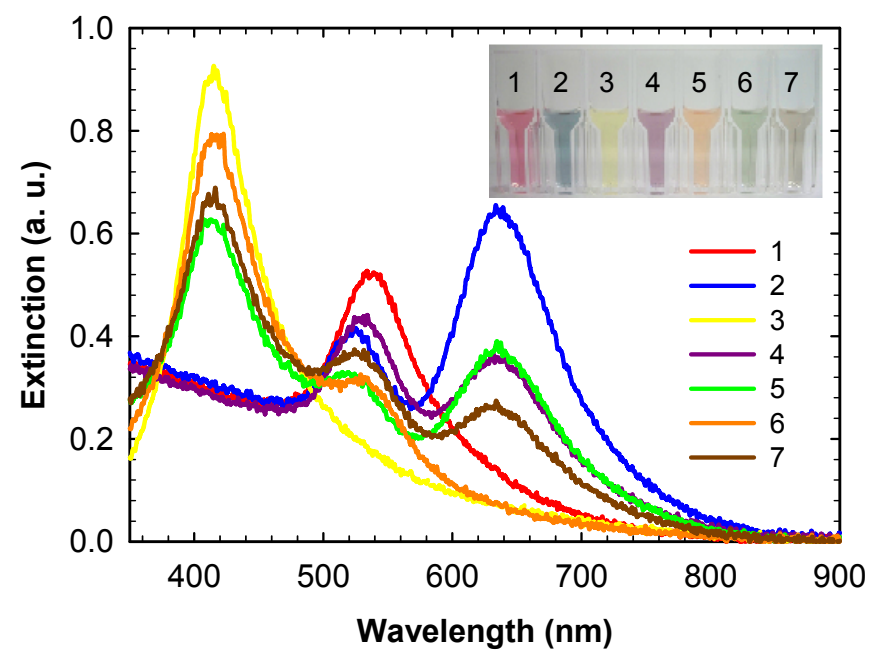

Figure 3. Plasmonic nanogels with tuned optical properties. 1) $20 \mathrm{ppm} 50 \mathrm{~nm}$ Au nanosphere (red). 2) $20 \mathrm{ppm} \mathrm{Au}$ nanorod with an aspect ratio of 2.3 (blue). 3) $20 \mathrm{ppm} 35 \mathrm{~nm}$ Ag nanosphere (yellow). 4) $10 \mathrm{ppm}$ Au nanosphere + $10 \mathrm{ppm} \mathrm{Au}$ nanorod (purple). 5) $25 \mathrm{ppm} \mathrm{Ag} \mathrm{nanosphere} \mathrm{+} 10 \mathrm{ppm}$ Au nanosphere (green). 6) $10 \mathrm{ppm} \mathrm{Au} \mathrm{nanorod}$ $+10 \mathrm{ppm} \mathrm{Ag} \mathrm{nanosphere} \mathrm{(orange).} \mathrm{7)} 15 \mathrm{ppm} \mathrm{Ag} \mathrm{nanosphere} \mathrm{+} 5 \mathrm{ppm}$ Au nanosphere $+10 \mathrm{ppm}$ Au nanorod (brown). Nanoparticles are distributed in the wormlike micellar solution of $100 \mathrm{mM} \mathrm{CTAB} / 100 \mathrm{mM} \mathrm{NaNO}$.

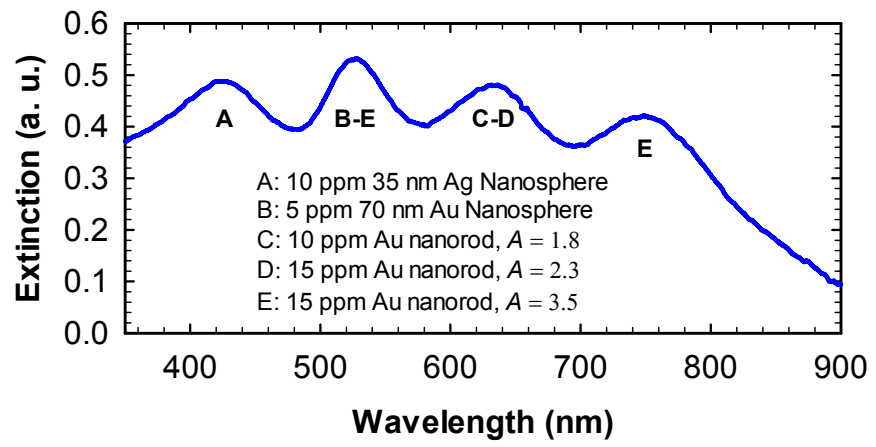

Figure 4. A multicomponent plasmonic nanogel with a relatively uniform extinction spectrum over the visible range. Five different nanoparticles (A-E) are suspended in the nanogel. Nanoparticles are distributed in the wormlike micellar solution of $100 \mathrm{mM} \mathrm{CTAB} / 100 \mathrm{mM} \mathrm{NaNO}_{3}$.

\section{RHEOLOGY AND STRUCTURE OF PLASMONIC NANOGELS}

PNGs are promising materials for optofluidics, not only because they have tunable optical properties, but also due to their low viscosity that allows it to flow at room temperature. To decipher the flow behavior and structural properties of the PNGs, we performed rheological measurements. A TA Instruments AR-G2 rheometer was used for this purpose. 100 $\mathrm{mM} \mathrm{CTAB} / 100 \mathrm{mM} \mathrm{NaNO}_{3}$ samples with $0,0.01,0.05,0.1$ and $0.2 w t . \%$ Ag nanoparticles were prepared and studied under steady shear flow rate from $1 \mathrm{~s}^{-1}$ to $1000 \mathrm{~s}^{-1}$. The neat WLM solution (no nanoparticles) exhibited a plateau at low shear rates and shear thinning at shear rate above $100 \mathrm{~s}^{-1}$. The decrease in viscosity at sufficiently large shear rate is due to flow alignment and breakage of the network. The shear rate $\dot{\gamma}^{*}$ at which shear thinning manifests can be used to estimate the relaxation time $\lambda_{\mathrm{r}}=\dot{\gamma}^{*-1}$. The relaxation times and zero shear viscosities $\left(\eta_{0}\right)$ were obtained by fitting the Carreau model to shear viscosity. ${ }^{32}$ With $0.01 \mathrm{wt} . \%$ nanoparticle added, the zero shear viscosity increases from 0.04 to $0.09 \mathrm{~Pa} \cdot \mathrm{s}$. The increase in the zero shear viscosity for samples with Ag NPs was much greater than what the Einstein equation for viscosity of dilute suspensions would predict. This is indicative of the formation of micelle-nanoparticle 
junctions. Significant rheological changes occured upon the addition of $0.2 w t . \%$ nanoparticles. Besides the increase in $\eta_{0}$, the onset of shear thinning shifts to a lower shear rate, which is indicative of a longer relaxation time.

Nanoparticles materially participating in the WLM network can enhance the network viscoelasticity leading to a substantial retardation of the nanoparticle mobility. Figure 6 shows the storage and loss moduli $G$ ' and $G$ ' of PNGs as a function of imposed frequency ranging from 0.1 to $100 \mathrm{~Hz} .100 \mathrm{mM} \mathrm{CTAB}$ and $100 \mathrm{mM} \mathrm{NaNO} \mathrm{WLM}_{3}$ solution with $0.01 \mathrm{wt} . \% \mathrm{Ag}$ nanoparticles did not exhibit any viscoelasticity. With the addition of $0.20 \mathrm{wt} . \%$ the sample was noticeably viscoelastic. Another method to enhance viscoelasticity of the PNGs is by introducing salt into the gel. As shown in Figure 6, with $200 \mathrm{mM} \mathrm{NaNO}_{3}$ and only $0.01 \mathrm{wt} \% \mathrm{Ag}$, the sample also exhibited viscoelasticity. The increase in $G_{0}$ observed upon the addition of the nanoparticles or salt can be interpreted as a commensurate increase in the network density $\rho=G_{0} / k_{\mathrm{b}} T$ that is caused by the formation of NP-mediated network junctions. The network mesh size of each sample can be estimated as $\left(k_{\mathrm{b}} T / G_{0}\right)^{1 / 3}$. The mesh size decreased from $82 \mathrm{~nm}$ to $55 \mathrm{~nm}$ as nanoparticle concentration increases from 0.01 to $0.20 \mathrm{wt} . \%$. These results provide a promising road map for using nanoparticle or salt as a means to control plasmonic nanogel flow behavior for new applications in plasmonics, micro/nanofluidics-based sensing and biology. ${ }^{32}$

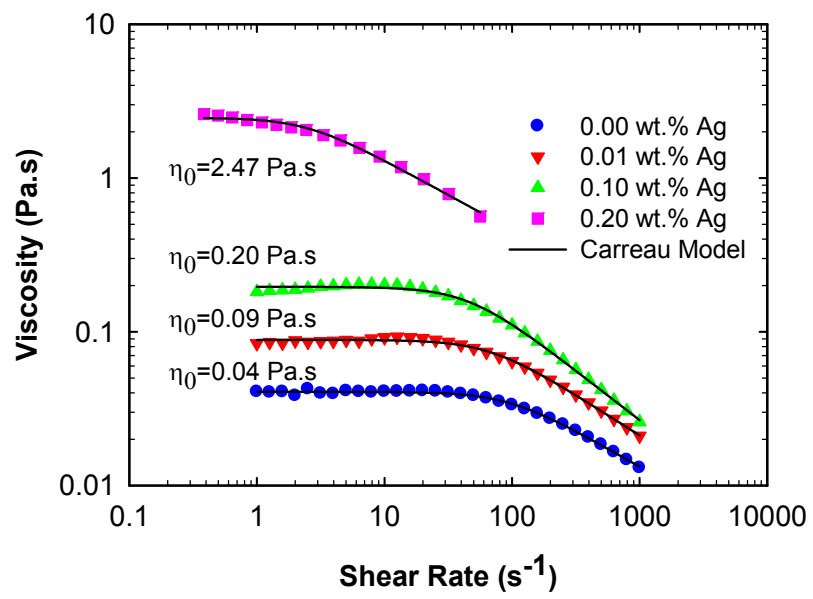

Figure 5. Steady state viscosity of plasmonic nanogels of $100 \mathrm{mM} \mathrm{CTAB}$ and $100 \mathrm{mM} \mathrm{NaNO}_{3}$ as a function of $\mathrm{Ag}$ nanoparticle concentration. Lines represent fits to the Carreau model.

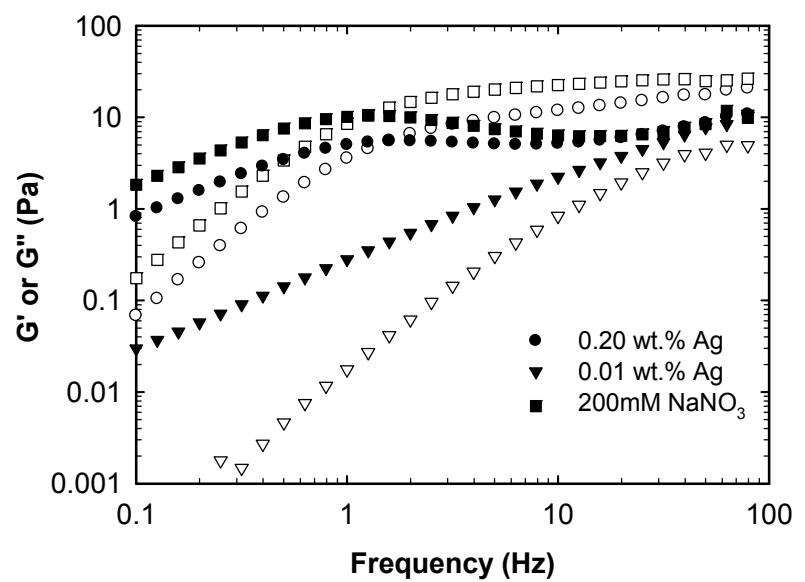

Figure 6. Linear viscoelastic spectrum of plasmonic nanogels (Storage modulus, $G$, open symbols and loss modulus, G', closed symbols). Sample I $(\bullet): 100 \mathrm{mM} \mathrm{CTAB}+100 \mathrm{mM} \mathrm{NaNO} 3+0.20 \mathrm{wt} . \% \mathrm{Ag}$. Sample II ( $\mathbf{\nabla})$ : $100 \mathrm{mM} \mathrm{CTAB}+100 \mathrm{mM} \mathrm{NaNO}_{3}+0.01$ wt.\% Ag. Sample III (匹): $100 \mathrm{mM} \mathrm{CTAB}+200 \mathrm{mM} \mathrm{NaNO}_{3}+0.01$ wt.\% Ag. 


\section{CONCLUSION}

Nanoparticles in solution are unstable and tend to agglomerate leading to phase separation, which limits the application of nanoparticle in fluidic systems. In this study, we design and synthesize multicomponent plasmonic nanogels by the self-assembly of different types metal nanoparticles with wormlike micelles in an aqueous solution. The nanogels have a long shelf life ( weeks) and exceptional color uniformity. The optical properties of the gels can be easily tuned by varying the type, shape and/or composition of nanoparticles. Nanogels with localized, multiple wavelength and broadband optical properties are synthesized in this work. Both nanoparticle and salt concentration have significant influence on the zero viscosity and viscoelasticity of the plasmonic nanogels. Their relatively low viscosity allows them to flow at room temperature. Thus, plasmonic nanogels combine the tunable optical properties and flow behavior. They are potentially suitable for optofluidic systems. Further, they can be processed by dip or spin coating onto substrates to produce thin films and interfaces that can be applied in photovoltaic devices and bio-molecular detection.

\section{ACKNOWLEDGMENTS}

This work is sponsored by the National Science Foundation through Grant CBET 1049454. Syracuse University has filed a provisional patent based on the results reported in this article.

\section{REFERENCES}

[1] Barnes, W. L., Dereux, A., and Ebbesen, T. W., "Surface plasmon subwavelength optics," Nature, 424(6950), 824830 (2003).

[2] Maier, S. A., and Atwater, H. A., "Plasmonics: Localization and guiding of electromagnetic energy in metal/dielectric structures," Journal of Applied Physics, 98, 011101 (2005).

[3] Aubry, A., Lei, D. Y., Fern·ndez-DomÌnguez, A. I., Sonnefraud, Y., Maier, S. A., and Pendry, J., "Plasmonic LightHarvesting Devices over the Whole Visible Spectrum," Nano Letters, 10, 2574-2579 (2010).

[4] Steele, J. M., Grandy, N. K., Nordlander, P., and Halas, N. J., [Surface Plasmon Nanophotonics] Springer, Dordrecht, Netherlands(2007).

[5] Bohren, C. F., and Huffman, D. R., “Absorption and scattering of light by small particles," Research supported by the University of Arizona and Institute of Occupational and Environmental Health. New York, Wiley-Interscience, 1983, 541 p., 1, (1983).

[6] Gonzalez, A., and Noguez, C., "Influence of morphology on the optical properties of metal nanoparticles," Journal of Computational and Theoretical Nanoscience, 4(2), 231-238 (2007).

[7] Daniel, M. C., and Astruc, D., "Gold nanoparticles: assembly, supramolecular chemistry, quantum-size-related properties, and applications toward biology, catalysis, and nanotechnology," Chemical Reviews, 104(1), 293-346 (2004).

[8] Wang, H., Brandl, D. W., Le, F., Nordlander, P., and Halas, N. J., "Nanorice: A hybrid plasmonic nanostructure," Nano Letters, 6(4), 827-832 (2006).

[9] Sherry, L. J., Chang, S. H., Schatz, G. C., Van Duyne, R. P., Benjamin, J., and Xia, Y., "Localized surface plasmon resonance spectroscopy of single silver nanocubes," Nano Letters, 5(10), 2034-2038 (2005).

[10] Tian, Z. Q., "Surface enhanced Raman spectroscopy: advancements and applications," Journal of Raman Spectroscopy, 36(6 7), 466-470 (2005).

[11] Haes, A. J., Chang, L., Klein, W. L., and Van Duyne, R. P., "Detection of a biomarker for Alzheimer's disease from synthetic and clinical samples using a nanoscale optical biosensor," Journal of the American Chemical Society, 127(7), 2264-2271 (2005).

[12] Jain, P., Huang, X., El-Sayed, I., and El-Sayed, M., "Review of Some Interesting Surface Plasmon Resonanceenhanced Properties of Noble Metal Nanoparticles and Their Applications to Biosystems," Plasmonics, 2(3), 107-118 (2007).

[13] Gobin, A. M., Lee, M. H., Halas, N. J., James, W. D., Drezek, R. A., and West, J. L., "Near-infrared resonant nanoshells for combined optical imaging and photothermal cancer therapy," Nano Letters, 7(7), 1929-1934 (2007). 
[14] Pillai, S., Catchpole, K., Trupke, T., and Green, M., "Surface plasmon enhanced silicon solar cells," Journal of Applied Physics, 101, 093105 (2007).

[15] Torkamani, S., Wani, S., Tang, Y., and Sureshkumar, R., "Plasmon-enhanced microalgal growth in miniphotobioreactors," Applied Physics Letters, 97, 043703 (2010).

[16] Liu, G. L., Kim, J., Lu, Y., and Lee, L. P., "Optofluidic control using photothermal nanoparticles," Nature materials, 5(1), 27-32 (2005).

[17] Psaltis, D., Quake, S. R., and Yang, C., "Developing optofluidic technology through the fusion of microfluidics and optics," Nature, 442(7101), 381-386 (2006).

[18] Atwater, H. A., and Polman, A., "Plasmonics for improved photovoltaic devices," Nature materials, 9(3), 205-213 (2010).

[19] Lee, S. K., Kim, S. H., Kang, J. H., Park, S. G., Jung, W. J., Yi, G. R., and Yang, S. M., "Optofluidics technology based on colloids and their assemblies," Microfluidics and Nanofluidics, 4(1), 129-144 (2008).

[20] Gehr, R. J., and Boyd, R. W., "Optical properties of nanostructured optical materials," Chemistry of materials, 8(8), 1807-1819 (1996).

[21] Garcia, H., Trice, J., Kalyanaraman, R., and Sureshkumar, R., "Self-consistent determination of plasmonic resonances in ternary nanocomposites," Physical Review B, 75(4), 045439 (2007).

[22] Hertel, G., and Hoffmann, H., "Lyotropic nematic phases of double chain surfactants," Trends in Colloid and Interface Science II, 123-131 (1988).

[23] Nikoobakht, B., and El-Sayed, M. A., "Evidence for bilayer assembly of cationic surfactants on the surface of gold nanorods," Langmuir, 17(20), 6368-6374 (2001).

[24] Nettesheim, F., Liberatore, M. W., Hodgdon, T. K., Wagner, N. J., Kaler, E. W., and Vethamuthu, M., "Influence of Nanoparticle Addition on the Properties of Wormlike Micellar Solutions," Langmuir, 24(15), 7718-7726 (2008).

[25] Helgeson, M. E., Hodgdon, T. K., Kaler, E. W., Wagner, N. J., Vethamuthu, M., and Ananthapadmanabhan, K., "Formation and Rheology of Viscoelastic iDouble Networksî in Wormlike Micelle- Nanoparticle Mixtures," Langmuir, 26(11), 8049-8060 (2010).

[26] Hohensu, A., Leitner, A., and Aussenegg, F. R., [Surface plasmon nanophotonics] Springer, Dordrecht, Netherlands(2007).

[27] Mayer, K. M., and Hafner, J. H., "Localized surface plasmon resonance sensors," Chemical Reviews, 111(6), 3828 (2011).

[28] Cong, T., Wani, S. N., Paynter, P. A., and Sureshkumar, R., "Structure and optical properties of self-assembled multicomponent plasmonic nanogels," Applied Phsics Letters, 99, 043112 (2011).

[29] Lu, Y., Liu, G. L., Kim, J., Mejia, Y. X., and Lee, L. P., "Nanophotonic crescent moon structures with sharp edge for ultrasensitive biomolecular detection by local electromagnetic field enhancement effect," Nano Letters, 5(1), 119124 (2005).

[30] Nehl, C. L., Liao, H., and Hafner, J. H., "Optical properties of star-shaped gold nanoparticles," Nano Letters, 6(4), 683-688 (2006).

[31] Schwartzberg, A. M., and Zhang, J. Z., "Novel Optical Properties and Emerging Applications of Metal Nanostructures," The Journal of Physical Chemistry C, 112(28), 10323-10337 (2008).

[32] Macosko, C. W., and Larson, R. G., [Rheology: principles, measurements, and applications] Wiley-Vch New York, (1994). 